\section{Blue Rubber Bleb Nevus Syndrome: Small-Bowel Lesions Diagnosed by Capsule Endoscopy}

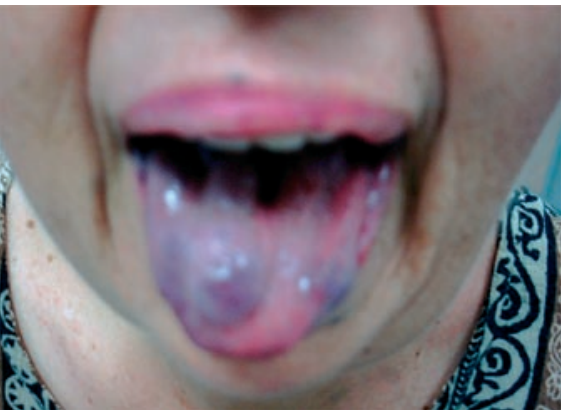

Figure 1 A 57-year-old woman was admitted to hospital due to chronic iron-deficiency anemia (<10 g\%). She denied having observed any overt gastrointestinal bleeding; her hemoglobin level at admission was $6.4 \mathrm{~g} \%$. There were multiple hemangiomas on her back and limbs, in her mouth, and underneath and on top of the tongue. Abdominal computed tomography showed a number of various-sized lesions in the liver. An isotope-marked red blood cell scan showed a concentration of lesions in the liver and mediastinum. Colonoscopy was normal. Esophagogastroduodenoscopy revealed two vascular lesions on the greater curvature of the stomach, with no active bleeding.

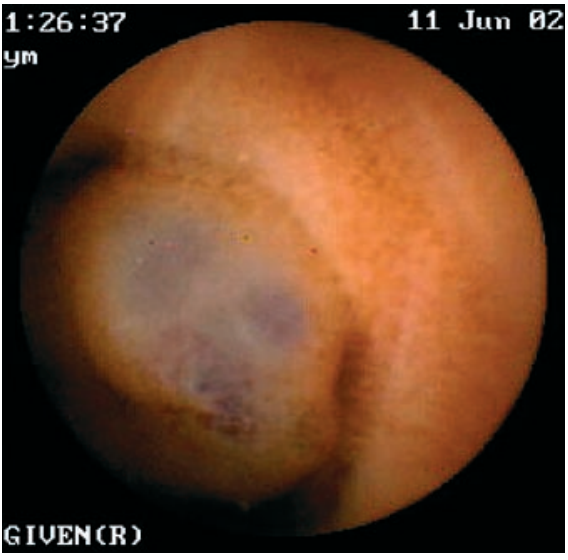

Figure 2 It was therefore decided to carry out a capsule endoscopy, which revealed a number of various-sized vascular lesions in the jejunum. The largest lesion was $2 \mathrm{~cm}$ in diameter, without any active bleeding.
L. Fish, Z. Fireman, Y. Kopelman,

\section{A. Sternberg}

Dept. of Gastroenterology, Hillel-Yaffe Medical Center, Hadera, and Bruce Rappaport Faculty of Medicine, The Technion, Haifa, Israel

\section{Acknowledgments}

Z. Fireman is a member of the medical advisory board of Given Imaging, Ltd. We are grateful to Carmela Reisler for editorial assistance.

Corresponding Author

\section{Z. Fireman, M.D.}

Dept. of Gastroenterology Hillel-Yaffe Medical Center

P.O. Box 169

Hadera 38100

Israel

Fax: +972-4-6304408

E-mail: fireman@hillel-yaffe.health.gov.il 